INPLASY

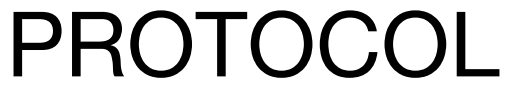

To cite: Hou et al. Efficacy and safety of sigmoidectomy for the treatment of sigmoid colon cancer: a protocol of systematic review. Inplasy protocol 202070056. doi: 10.37766/inplasy2020.7.0056

Received: 14 July 2020

Published: 14 July 2020

Corresponding author: Qing Li

qikepai48755@21cn.com

Author Affiliation:

The First Hospital of Yulin

Support: SXKRDP

(No. 2017SF-207)

Review Stage at time of this submission: The review has not yet started.

Conflicts of interest: No.

\section{Efficacy and safety of sigmoidectomy for the treatment of sigmoid colon cancer: a protocol of systematic review}

Hou, HP1; Li, Q2 .

Review question / Objective: Is sigmoidectomy effective and safe for the treatment of sigmoid colon cancer (SCC)? Condition being studied: Sigmoidectomy; sigmoid colon cancer.

Information sources: Electronic databases search - We will search the following databases from inception to July 31, 2020: MEDLINE, EMBASE, Cochrane Library, CINAHL, PsycINFO, Scopus, OpenGrey, Chinese Biomedical Literature Database, and China National Knowledge In frastructure. No language and publication status limitations will be imposed to all above electronic databases. We will build a detailed search strategy for Cochrane Library in table 1. In addition, we will also adapt similar search strategies to any other electronic databases. Search for other resources - Aside from above electronic databases, we will also search conference proceedings, dissertations, and reference list of associated reviews.

INPLASY registration number: This protocol was registered with the International Platform of Registered Systematic Review and Meta-Analysis Protocols (INPLASY) on 14 July 2020 and was last updated on 14 July 2020 (registration number INPLASY202070056).

\section{INTRODUCTION}

Review question / Objective: Is sigmoidectomy effective and safe for the treatment of sigmoid colon cancer (SCC)?

Condition being studied: Sigmoidectomy; sigmoid colon cancer.

\section{METHODS}

Participant or population: Inclusion criteria for study participants will be all eligible patients who were diagnosed as SCC. No limitations will be applied in terms of country, ethnicity, and educational background. 
Intervention: In the experimental group, intervention to be utilized is sigmoidectomy alone.

Comparator: In the control group, any treatment management could be used, such as radiotherapy, chemotherapy, but not sigmoidectomy.

Study designs to be included: All relevant randomized controlled trials (RCTs) of sigmoidectomy in treating SCC will be included.

Eligibility criteria: All relevant RCTs of sigmoidectomy in treating SCC will be included without restrictions of language and publication status.

Information sources: Electronic databases search - We will search the following databases from inception to July 31, 2020: MEDLINE, EMBASE, Cochrane Library, CINAHL, PsycINFO, Scopus, OpenGrey, Chinese Biomedical Literature Database, and China National Knowledge In $\neg$ frastructure. No language and publication status limitations will be imposed to all above electronic databases. We will build a detailed search strategy for Cochrane Library in table 1. In addition, we will also adapt similar search strategies to any other electronic databases. Search for other resources - Aside from above electronic databases, we will also search conference proceedings, dissertations, and reference list of associated reviews.

Main outcome(s): Outcomes include overall survival, pathological complete response, progression-free survival, recurrence-free survival, and any complication.

Data management: Two researchers will independently extract all relevant data from included studies using a standardized data extraction sheet. Any deviations between two researchers will be coped with a third researcher through consultation. The extracted information consist of study title, first author, country, time of publication, characteristics of patients, study setting, randomization, binding, allocation, concealment, sample size, details of interventions, controls, follow-up, outcome indicators, study results, adverse events, and funding information. If we identify any missing or unclear data, we will contact primary author to request them by email.

Quality assessment / Risk of bias analysis: Two researchers will independently appraise the risk of bias assessment for each qualified study using Cochrane risk of bias tool based on the guidelines of Cochrane Handbook for Systematic Reviews of Interventions. It covers 7 aspects, and each item is further referred as low, unclear or high risk of bias. Any different opinions between two researchers will be disentangled by a third researcher through discussion.

Strategy of data synthesis: RevMan $\mathbf{5 . 3}$ software will be employed for statistical analysis in this study. Meta-analysis will be undertaken if we identified low level of heterogeneity, and sufficient data are collected on the similar characteristics of study and patient, treatments, controls, and outcome records. Otherwise, if high level of heterogeneity is found, we will perform subgroup analysis to detect the possible reasons for such situation. Moreover, we will carry out narrative summary for the study results by reporting detailed written commentary to present the target patient characteristics, study findings, types of treatments and controls, and outcomes.

Subgroup analysis: We will implement subgroup analysis according to the different characteristics of study or patient, intervention, comparators, and outcomes.

Sensibility analysis: We will preside over sensitivity analysis to explore the robustness of outcome results by excluding low quality studies.

Country(ies) involved: China.

Keywords: Sigmoid colon cancer; sigmoidectomy; efficacy; safety.

Contributions of each author:

Author 1 - Hua-ping Hou.

Author 2 - Qing Li. 